\title{
Singular Solutions of Hessian Elliptic Equations in Five Dimensions
}

\author{
Nikolai Nadirashvili, Serge Vlăduţ†
}

October 10, 2018

\section{Introduction}

In this paper we study a class of fully nonlinear second-order elliptic equations of the form

$$
F\left(D^{2} u\right)=0
$$

defined in a domain of $\mathbb{R}^{n}$. Here $D^{2} u$ denotes the Hessian of the function $u$. We assume that $F$ is a Lipschitz function defined on the space $S^{2}\left(\mathbb{R}^{n}\right)$ of $n \times n$ symmetric matrices satisfying the uniform ellipticity condition, i.e. there exists a constant $C=C(F) \geq 1$ (called an ellipticity constant) such that

$$
C^{-1}\|N\| \leq F(M+N)-F(M) \leq C\|N\|
$$

for any non-negative definite symmetric matrix $N$; if $F \in C^{1}\left(S^{2}\left(\mathbb{R}^{n}\right)\right)$ then this condition is equivalent to

$$
\frac{1}{C^{\prime}}|\xi|^{2} \leq F_{u_{i j}} \xi_{i} \xi_{j} \leq C^{\prime}|\xi|^{2}, \forall \xi \in \mathbb{R}^{n} .
$$

Here, $u_{i j}$ denotes the partial derivative $\partial^{2} u / \partial x_{i} \partial x_{j}$. A function $u$ is called a classical solution of (1) if $u \in C^{2}(\Omega)$ and $u$ satisfies (1.1). Actually, any classical solution of (1.1) is a smooth $\left(C^{\alpha+3}\right)$ solution, provided that $F$ is a smooth $\left(C^{\alpha}\right)$ function of its arguments.

For a matrix $S \in S^{2}\left(\mathbb{R}^{n}\right)$ we denote by $\lambda(S)=\left\{\lambda_{i}: \lambda_{1} \leq \ldots \leq \lambda_{n}\right\} \in \mathbb{R}^{n}$ the (ordered) set of eigenvalues of the matrix $S$. Equation (1.1) is called a Hessian equation ([T1],[T2] cf. [CNS]) if the function $F(S)$ depends only on the eigenvalues $\lambda(S)$ of the matrix $S$, i.e., if

$$
F(S)=f(\lambda(S)),
$$

*CMI, 39, rue F. Joliot-Curie, 13453 Marseille FRANCE, nicolas@cmi.univ-mrs.fr

${ }^{\dagger}$ IML, Luminy, case 907, 13288 Marseille Cedex FRANCE and IITP RAS, B.Karetnyi,9, Moscow, RUSSIA, vladut@iml.univ-mrs.fr 
for some function $f$ on $\mathbb{R}^{n}$ invariant under permutations of the coordinates.

In other words the equation (1.1) is called Hessian if it is invariant under the action of the group $O(n)$ on $S^{2}\left(\mathbb{R}^{n}\right)$ :

$$
\forall O \in O(n), F\left({ }^{t} O \cdot S \cdot O\right)=F(S) .
$$

The Hessian invariance relation (1.3) implies the following:

(a) $F$ is a smooth (real-analytic) function of its arguments if and only if $f$ is a smooth (real-analytic) function.

(b) Inequalities (1.2) are equivalent to the inequalities

$$
\frac{\mu}{C_{0}} \leq f\left(\lambda_{i}+\mu\right)-f\left(\lambda_{i}\right) \leq C_{0} \mu, \forall \mu \geq 0,
$$

$\forall i=1, \ldots, n$, for some positive constant $C_{0}$.

(c) $F$ is a concave function if and only if $f$ is concave.

Well known examples of the Hessian equations are Laplace, Monge-Ampère, Bellman, Isaacs and Special Lagrangian equations.

Bellman and Isaacs equations appear in the theory of controlled diffusion processes, see $[\mathrm{F}]$. Both are fully nonlinear uniformly elliptic equations of the form (1.1). The Bellman equation is concave in $D^{2} u \in S^{2}\left(\mathbb{R}^{n}\right)$ variables. However, Isaacs operators are, in general, neither concave nor convex. In a simple homogeneous form the Isaacs equation can be written as follows:

$$
F\left(D^{2} u\right)=\sup _{b} \inf _{a} L_{a b} u=0,
$$

where $L_{a b}$ is a family of linear uniformly elliptic operators of type

$$
L=\sum a_{i j} \frac{\partial^{2}}{\partial x_{i} \partial x_{j}}
$$

with an ellipticity constant $C>0$ which depends on two parameters $a, b$.

Consider the Dirichlet problem

$$
\left\{\begin{array}{cl}
F\left(D^{2} u, D u, u, x\right)=0 & \text { in } \Omega \\
u=\varphi & \text { on } \partial \Omega,
\end{array}\right.
$$

where $\Omega \subset \mathbb{R}^{n}$ is a bounded domain with a smooth boundary $\partial \Omega$ and $\varphi$ is a continuous function on $\partial \Omega$.

We are interested in the problem of existence and regularity of solutions to the Dirichlet problem (1.6) for Hessian equations and Isaacs equation. The problem (1.6) has always a unique viscosity (weak) solution for fully nonlinear elliptic equations (not necessarily Hessian equations). The viscosity solutions satisfy the equation (1.1) in a weak sense, and the best known interior regularity $([\mathrm{C}],[\mathrm{CC}],[\mathrm{T} 3])$ for them is $C^{1+\epsilon}$ for some $\epsilon>0$. For more details see 
$[\mathrm{CC}],[\mathrm{CIL}]$. Until recently it remained unclear whether non-smooth viscosity solutions exist. In the recent papers [NV1], [NV2], [NV3], [NV4] the authors first proved the existence of non-classical viscosity solutions to a fully nonlinear elliptic equation, and then of singular solutions to Hessian uniformly elliptic equation in all dimensions beginning from 12. Those papers use the functions

$$
w_{12, \delta}(x)=\frac{P_{12}(x)}{|x|^{\delta}}, w_{24, \delta}(x)=\frac{P_{24}(x)}{|x|^{\delta}}, \delta \in[1,2[,
$$

with $P_{12}(x), P_{24}(x)$ being cubic forms as follows:

$$
P_{12}(x)=\operatorname{Re}\left(q_{1} q_{2} q_{3}\right), x=\left(q_{1}, q_{2}, q_{3}\right) \in \mathbb{H}^{3}=\mathbb{R}^{12},
$$

$\mathbb{H}$ being Hamiltonian quaternions,

$$
P_{24}(x)=\operatorname{Re}\left(\left(o_{1} \cdot o_{2}\right) \cdot o_{3}\right)=\operatorname{Re}\left(o_{1} \cdot\left(o_{2} \cdot o_{3}\right)\right), x=\left(o_{1}, o_{2}, o_{3}\right) \in \mathbb{O}^{3}=\mathbb{R}^{24}
$$

(1) being the algebra of Caley octonions.

Finally, the paper [NTV] gives a construction of non-smooth viscosity solution in 5 dimensions which is order 2 homogeneous, also for Hessian equations, the function

$$
w_{5}(x)=\frac{P_{5}(x)}{|x|},
$$

being such solution for the Cartan minimal cubic

$$
P_{5}(x)=x_{1}^{3}+\frac{3 x_{1}}{2}\left(z_{1}^{2}+z_{2}^{2}-2 z_{3}^{2}-2 x_{2}^{2}\right)+\frac{3 \sqrt{3}}{2}\left(x_{2} z_{1}^{2}-x_{2} z_{2}^{2}+2 z_{1} z_{2} z_{3}\right)
$$

in 5 dimensions.

However, the methods of $[\mathrm{NTV}]$ does not work for the function $w_{5, \delta}(x)=$ $P_{5}(x) /|x|^{\delta}, \delta>1$, and thus does not give singular (i.e. not in $C^{1,1}$ ) viscosity solutions to fully nonlinear equations in 5 dimensions.

In the present paper we fill the gap and prove

\section{Theorem 1.1.}

The function

$$
w_{5, \delta}(x)=P_{5}(x) /|x|^{1+\delta}, \delta \in[0,1[
$$

is a viscosity solution to a uniformly elliptic Hessian equation (1.1) with a smooth functional $F$ in a unit ball $B \subset \mathbb{R}^{5}$ for the isoparametric Cartan cubic form

$$
P_{5}(x)=x_{1}^{3}+\frac{3 x_{1}}{2}\left(z_{1}^{2}+z_{2}^{2}-2 z_{3}^{2}-2 x_{2}^{2}\right)+\frac{3 \sqrt{3}}{2}\left(x_{2} z_{1}^{2}-x_{2} z_{2}^{2}+2 z_{1} z_{2} z_{3}\right)
$$

with $x=\left(x_{1}, x_{2}, z_{1}, z_{2}, z_{3}\right)$.

In particular one gets the optimality of the interior $C^{1, \alpha}$-regularity of viscosity solutions to fully nonlinear equations in dimensions 5 and more; note also 
that all previous constructions give only Lipschitz Hessian functional $F$. Let us recall that in the paper [NV5] it is proven that there is no order 2 homogenous solutions to elliptic equations in 4 dimensions which suggests strongly that in 4 (and less) dimensions there is no homogenous non-classical solutions to uniformly elliptic equations.

As in [NV3] we get also that $w_{5, \delta}(x), \delta \in[0,1[$ is a viscosity solution to a uniformly elliptic Isaacs equation:

\section{Corollary 1.2.}

The function

$$
w_{5, \delta}(x)=P_{5}(x) /|x|^{1+\delta}, \delta \in[0,1[
$$

is a viscosity solution to a uniformly elliptic Isaacs equation (1.4) in a unit ball $B \subset \mathbb{R}^{5}$.

The rest of the paper is organized as follows: in Section 2 we recall some necessary preliminary results and we prove our main results in Section 3. The proof in Section 3 extensively uses MAPLE but is completely rigorous.

\section{Preliminary results}

Let $w=w_{n}$ be an odd homogeneous function of order $2-\delta, 0 \leq \delta<1$, defined on a unit ball $B=B_{1} \subset \mathbb{R}^{n}$ and smooth in $B \backslash\{0\}$. Then the Hessian of $w$ is homogeneous of order $-\delta$.

Define the map

$$
\Lambda: B \longrightarrow \lambda(S) \in \mathbb{R}^{n} .
$$

$\lambda(S)=\left\{\lambda_{i}: \lambda_{1} \leq \ldots \leq \lambda_{n}\right\} \in \mathbb{R}^{n}$ being the (ordered) set of eigenvalues of the matrix $S=D^{2} w$. Denote $\Sigma_{n}$ the permutation group of $\{1, \ldots, n\}$. For any $\sigma \in$ $\Sigma_{n}$, let $T_{\sigma}$ be the linear transformation of $\mathbb{R}^{n}$ given by $x_{i} \mapsto x_{\sigma(i)}, i=1, \ldots, n$.

Let $a, b \in B$. Denote by $\mu_{1}(a, b) \leq \ldots \leq \mu_{n}(a, b)$ the eigenvalues of $\left(D^{2} w(a)-\right.$ $\left.D^{2} w(b)\right)$.

Lemma 2.1. Assume that for a smooth function $g: U \longrightarrow \mathbb{R}$ where the domain $U$ contains

$$
M:=\bigcup_{\sigma \in \Sigma_{n}} T_{\sigma} \Lambda(B) \subset \mathbb{R}^{n}
$$

one has

$$
g_{\mid M}=0 .
$$

Assume also the condition

$$
\min _{i=1, \ldots, 5} \inf _{x \in M}\left\{\frac{\partial g}{\partial \lambda_{i}}(\lambda)\right\}>0 .
$$


Assume further that for any $a, b \in B$ either $\mu_{1}(a, b)=\ldots=\mu_{n}(a, b)=0$ or

$$
1 / C \leq-\frac{\mu_{1}(a, b)}{\mu_{n}(a, b)} \leq C,
$$

where $C$ is a positive constant (may be, depending on $M, g$ but not on $a, b$ ). If $\delta>0$ we assume additionally that $w$ changes sign in $B$. Then $w$ is a viscosity solution in $B$ of a uniformly elliptic Hessian equation (1.1) with a smooth $F$. Function $w$ is as well a solution to a uniformly elliptic Isaacs equation.

Proof. Denote for any $\theta>0$ by $K_{\theta} \subset \mathbb{R}^{n}$ the cone $\left\{\lambda \in \mathbb{R}^{n}, \lambda_{i} /|\lambda|>\theta\right\}$, and let $K_{\theta}^{*}$ be its dual cone. Let $x, y$ be orthogonal coordinates in $\mathbb{R}^{n}$ such that $x=\lambda_{1}+\ldots+\lambda_{n}$ and $y$ be the orthogonal complement of $x$. Denote by $p$ the orthogonal projection of $\mathbb{R}^{n}$ on subspace $y$. Denote

$$
\begin{gathered}
\Gamma=\{g=0\} \subset U, \\
G=p(\Gamma), \\
m=p(M) .
\end{gathered}
$$

From (2.1), (2.2) it follows that the surface $\Gamma$ is a graph of a smooth function $h$ defined on $G$. By $k_{\theta}$ we denote the function on $y$ which graph is the surface $\partial K_{\theta}^{*}$. We define the function $H(y)$ by

$$
H(y)=\inf _{z \in G}\left\{h(z)+k_{\theta}(y-z)\right\} .
$$

We fix a sufficiently small $\theta>0$. Then from (2.1), (2.2) it follows that $H=h$ on $G$. Denote by $J$ the graph of $H$. It is easy to show, see similar argument in [NV1], [NV3], that for any $a, b \in J, a \neq b$,

$$
1 / C \leq-\min _{i}\left(a_{i}-b_{i}\right) / \max _{i}\left(a_{i}-b_{i}\right) \leq C .
$$

Let $E$ be a smooth function in $\mathbb{R}^{n-1}$ with the support in a unit ball and with the integral being equal to 1 . Denote $E_{c}(y)=c^{-n+1} E(y / c), c>0$. Set

$$
H_{c}=H * E_{c} .
$$

Then $H_{c}$ will be a smooth function such that any two points $a, b$ on its graph will satisfy (2.3). Moreover $H_{c} \rightarrow H$ in $C\left(\mathbb{R}^{n}\right)$ as $c$ goes to 0 , and $H_{c} \rightarrow h$ in $C^{\infty}$ on compact subdomains of $G$. Thus for a sufficiently small $c>0$ we can easily modify function $H_{c}$ to a function $\widetilde{H}$ such that $\widetilde{H}$ will coincide with $h$ in a neighborhood of $m$, coincide with $H$ in the complement of $G$ and the points on the graph of $\widetilde{H}$ will still satisfy (2.3) possibly with a larger constant $C$. Define the function $F$ in $\mathbb{R}^{n}$ by

$$
F=x-\widetilde{H}(y) .
$$

Then $w$ is a solution in $\mathbb{R}^{n} \backslash\{0\}$ of a uniformly elliptic Hessian equation (1.1) with such defined nonlinearity $F$. As in [NV3], [NV4] it follows that $w$ is a 
viscosity solution of (1.1) in the whole space $\mathbb{R}^{n}$. In [NV3] we have shown that the equation (1.1) for the function $w$ can be rewritten in the form of the Isaacs equation. The lemma is proved.

We will apply this result to the function $w_{5, \delta}(x)=P_{5}(x) /|x|^{1+\delta}$.

Let then recall some facts from $[\mathrm{NTV}]$ about the Cartan cubic form $P_{5}(x)$.

\section{Lemma 2.2.}

The form $P_{5}(x)$ admits a three-dimensional automorphism group.

Indeed, one easily verifies that the orthogonal trasformations

$$
\begin{gathered}
A_{1}(\phi):=\left(\begin{array}{ccccc}
\frac{3 \cos (\phi)^{2}-1}{2} & \frac{\sqrt{3} \sin (\phi)^{2}}{2} & 0 & 0 & \frac{\sqrt{3} \sin (2 \phi)}{2} \\
\frac{\sqrt{3} \sin (\phi)^{2}}{2} & \frac{1+\cos (\phi)^{2}}{2} & 0 & 0 & \frac{-\sin (2 \phi)}{2} \\
0 & 0 & \cos (\phi) & \sin (\phi) & 0 \\
0 & 0 & -\sin (\phi) & \cos (\phi) & 0 \\
\frac{-\sqrt{3} \sin (2 \phi)}{2} & \frac{\sin (2 \phi)}{2} & 0 & 0 & \cos (2 \phi)
\end{array}\right) \\
A_{2}(\psi):=\left(\begin{array}{cccccc}
1 & 0 & 0 & 0 & 0 \\
0 & \cos (2 \psi) & 0 & -\sin (2 \psi) & 0 \\
0 & 0 & \cos (\psi) & 0 & -\sin (\psi) \\
0 & \sin (2 \psi) & 0 & \cos (2 \psi) & 0 \\
0 & 0 & \sin (\psi) & 0 & \cos (\psi)
\end{array}\right) \\
A_{3}(\theta):=\left(\begin{array}{cccccc}
\frac{3 \cos (\theta)^{2}-1}{2} & \frac{-\sqrt{3} \sin (\theta)^{2}}{2} & 0 & 0 & \frac{-\sqrt{3} \sin (2 \theta)}{2} \\
\frac{-\sqrt{3} \sin (\theta)^{2}}{2} & \frac{1+\cos (\theta)^{2}}{2} & 0 & 0 & \frac{-\sin (2 \theta)}{2} \\
0 & 0 & \cos (\theta) & -\sin (\theta) & 0 \\
0 & 0 & \sin (\theta) & \cos (\theta) & 0 \\
\frac{\sqrt{3} \sin (2 \theta)}{2} & \frac{\sin (2 \theta)}{2} & 0 & 0 & \cos (2 \theta)
\end{array}\right)
\end{gathered}
$$

do not change the value of $P_{5}(x)$.

\section{Lemma 2.3.}

Let $G_{P}$ be subgroup of $S O(5)$ generated by

$\left\{A_{1}(\phi), A_{2}(\psi), A_{3}(\theta):(\phi, \psi, \theta) \in \mathbb{R}^{3}\right\}$. Then the orbit $G_{P} S^{1}$ of the circle

$$
S^{1}=\{(\cos (\chi), 0, \sin (\chi), 0,0): \chi \in \mathbb{R}\} \subset S^{4}
$$

under the natural action of $G_{P}$ is the whole $S^{4}$.

We need also the following two simple algebraic results ( [NV3, Lemmas 2.2 and 4.1]):

Lemma 2.4. Let $A, B$ be two real symmetric matrices with the eigenvalues $\lambda_{1} \geq \lambda_{2} \geq \ldots \geq \lambda_{n}$ and $\lambda_{1}^{\prime} \geq \lambda_{2}^{\prime} \geq \ldots \geq \lambda_{n}^{\prime}$ respectively. Then for the eigenvalues $\Lambda_{1} \geq \Lambda_{2} \geq \ldots \geq \Lambda_{n}$ of the matrix $A-B$ we have

$$
\Lambda_{1} \geq \max _{i=1, \cdots, n}\left(\lambda_{i}-\lambda_{i}^{\prime}\right), \quad \Lambda_{n} \leq \min _{i=1, \cdots, n}\left(\lambda_{i}-\lambda_{i}^{\prime}\right) .
$$


Lemma 2.5. Let $\delta \in[0,1), W, \quad \bar{W} \in \mathbb{R}$ with $|W| \leq \frac{1}{3 \sqrt{3}},|\bar{W}| \leq \frac{1}{3 \sqrt{3}}$ and let $\mu_{1}(\delta) \geq \mu_{2}(\delta) \geq \mu_{3}(\delta)$ (resp., $\bar{\mu}_{1}(\delta) \geq \bar{\mu}_{2}(\delta) \geq \bar{\mu}_{3}(\delta)$ ) be the roots of the polynomial

$$
T^{3}+3 W(1+\delta) T^{2}+\left(3 W^{2}(1+\delta)^{2}-1\right) T+W(1-\delta)+W^{3}(1+\delta)^{3}
$$

(resp. of the polynomial

$$
\left.T^{3}+3 \bar{W}(1+\delta) T^{2}+\left(3 \bar{W}^{2}(1+\delta)^{2}-1\right) T+\bar{W}(1-\delta)+\bar{W}^{3}(1+\delta)^{3}\right) .
$$

Then for any $K>0$ verifying $|K-1|+|\bar{W}-W| \neq 0$ one has

$$
\frac{1-\delta}{5+\delta}=: \rho \leq \frac{\mu_{+}(K)}{-\mu_{-}(K)} \leq \frac{1}{\rho}=\frac{5+\delta}{1-\delta}
$$

where

$$
\begin{gathered}
\mu_{-}(K):=\min \left\{\mu_{1}(\delta)-K \bar{\mu}_{1}(\delta), \mu_{2}(\delta)-K \bar{\mu}_{2}(\delta), \mu_{3}(\delta)-K \bar{\mu}_{3}(\delta)\right\}, \\
\mu_{+}(K):=\max \left\{\mu_{1}(\delta)-K \bar{\mu}_{1}(\delta), \mu_{2}(\delta)-K \bar{\mu}_{2}(\delta), \mu_{3}(\delta)-K \bar{\mu}_{3}(\delta)\right\} .
\end{gathered}
$$

\section{Proofs}

Let $w_{5, \delta}=P_{5} /|x|^{1+\delta}, \delta \in[0,1[$. By Lemma 2.1 it is sufficient to prove the existence of a smooth function $g$ verifying the conditions (2.1) and (2.2). We beging with calculating the eigenvalues of $D^{2} w_{5, \delta}(x)$. More precisely, we need

Lemma 3.1.

Let $x \in S^{4}$, and let $x \in G_{P}(p, 0, q, 0,0)$ with $p^{2}+q^{2}=1$. Then

$$
\operatorname{Spec}\left(D^{2} w_{5, \delta}(x)\right)=\left\{\mu_{1, \delta}, \mu_{2, \delta}, \mu_{3, \delta}, \mu_{4, \delta}, \mu_{5, \delta}\right\}
$$

for

$$
\begin{gathered}
\mu_{1, \delta}=\frac{p\left(p^{2} \delta+6-3 \delta\right)}{2}, \\
\mu_{2, \delta}=\frac{p\left(p^{2} \delta-3-3 \delta\right)+3 \sqrt{12-3 p^{2}}}{2}, \\
\mu_{3, \delta}=\frac{p\left(p^{2} \delta-3-3 \delta\right)-3 \sqrt{12-3 p^{2}}}{2}, \\
\mu_{4, \delta}=-\frac{p \delta(6-\delta)\left(3-p^{2}\right)+\sqrt{D(p, \delta)}}{4}, \\
\mu_{5, \delta}=-\frac{p \delta(6-\delta)\left(3-p^{2}\right)-\sqrt{D(p, \delta)}}{4},
\end{gathered}
$$

and

$$
D(p, \delta):=(6-\delta)(4-\delta)(2-\delta) \delta\left(p^{2}-3\right)^{2} p^{2}+144(\delta-2)^{2}>0 .
$$


The characteristic polynomial $F(S)$ of $D^{2} w$ is given by

$$
F(S)=S^{5}+a_{1, \delta} S^{4}+a_{2, \delta} S^{3}+a_{3, \delta} S^{2}+a_{4, \delta} S+a_{5, \delta}
$$

for

$$
\begin{gathered}
a_{1, \delta}=\frac{(\delta+1)(\delta-8) b}{2}, \\
a_{2, \delta}=\frac{(\delta+1)\left(21 \delta+13-4 \delta^{2}\right) b^{2}}{4}+9\left(2 \delta-\delta^{2}-4\right), \\
a_{3, \delta}=\frac{\left(6 \delta^{2}-31 \delta-1\right)(\delta+1)^{2} b^{3}}{8}+\frac{27\left(4 \delta-2 \delta^{2}+5+\delta^{3}\right)}{2}, \\
a_{4, \delta}=\frac{(2 \delta-1)(5-\delta)(\delta+1)^{2} b^{4}}{8}+\frac{9(\delta-1)\left(\delta^{2}-2 \delta+9\right)}{2}, \\
a_{5, \delta}=\frac{b(1-\delta)\left(b^{2}(\delta+1)^{3}+108(1-\delta)\right)\left(b^{2}(\delta+1)(\delta-5)+36(\delta-1)\right)}{32}, \\
\text { where } b:=p\left(p^{2}-3\right) .
\end{gathered}
$$

Note that the spectrum in this lemma is unordered one.

Proof of Lemma 3.1. Since $w_{5, \delta}$ is invariant under $G_{P}$, we can suppose that $x=(p, 0, q, 0,0)$. Then $w_{5, \delta}(x)=\frac{p\left(3-p^{2}\right)}{2}$ and we get by a brute force calculation:

$$
D^{2} w_{5, \delta}(x):=\left(\begin{array}{cc}
M_{1, \delta} & 0 \\
0 & M_{2, \delta}
\end{array}\right)
$$

being a block matrix with

$$
M_{1, \delta}:=\frac{1}{2}\left(\begin{array}{lll}
m_{1,1} & m_{1,2} & m_{1,3} \\
m_{1,2} & m_{2,2} & m_{2,3} \\
m_{1,3} & m_{2,3} & m_{3,3}
\end{array}\right),
$$

$$
\begin{aligned}
& m_{1,1}:=-(\delta+2) \delta p^{5}+(\delta+3) \delta p^{3}+(12-9 \delta) p \\
& m_{1,2}:=3 \sqrt{3} p\left(p^{2}-1\right) \delta \\
& \left.m_{1,3}:=-q\left((\delta+2) \delta p^{4}+3 \delta(1-\delta) p^{2}+3 \delta-6\right)\right) \\
& m_{2,2}:=\delta p^{3}-3(\delta+4) p \\
& m_{2,3}:=3 \sqrt{3} q\left(\delta p^{2}+2-\delta\right) \\
& m_{3,3}:=(\delta+2) \delta p^{5}+(5-4 \delta) \delta p^{3}-3(\delta-1)(2-\delta) p
\end{aligned}
$$

$$
M_{2, \delta}:=\frac{1}{2}\left(\begin{array}{cc}
\delta p^{3}+3(2-\delta) p & 6 \sqrt{3} q \\
6 \sqrt{3} q & \delta p^{3}-3(4+\delta) p
\end{array}\right)
$$

which gives for the characteristic polynomial $F(S)=F_{1}(S) \cdot F_{2}(S) \cdot F_{3}(S)$ where

$$
F_{1}(S):=S-\frac{p\left(p^{2} \delta+6-3 \delta\right)}{2}
$$




$$
\begin{gathered}
F_{2}(S)=S^{2}+\frac{\delta p\left(p^{2}-3\right)(\delta-6) S}{2}+\frac{(2-\delta)\left((\delta-6) \delta p^{6}+6(6-\delta) \delta^{2} p^{4}+9\left(\delta^{2}-6 \delta\right) p^{2}+36(\delta-2)\right)}{4} ; \\
F_{3}(S):=S^{2}+\left(3+3 \delta-\delta p^{2}\right) p S+\frac{\left(p^{2}-3\right)\left(\delta^{2} p^{4}-3 \delta^{2} p^{2}-6 \delta p^{2}+36\right)}{4}
\end{gathered}
$$

and the spectrum. Developing $F(S)$ we get the last formulas.

Corollary 3.1. Denote $\varepsilon=1-\delta$. The function $w$ verifies the following Hessian equation:

$$
\operatorname{det}\left(D^{2} w\right)=e_{5}(\Delta(w))^{5}+e_{3}(\Delta(w))^{3} S_{2}(w)+e_{1} \Delta(w) S_{4}(w)
$$

where

$$
\begin{gathered}
e_{5}=\frac{\varepsilon^{2}\left(168-5 \varepsilon^{4}-24 \varepsilon^{3}-56 \varepsilon\right)}{\left(\varepsilon^{2}+3\right)(\varepsilon+7)^{5}(\varepsilon-2)^{3}} \\
e_{3}=\frac{\varepsilon^{2}\left(2 \varepsilon^{2}+\varepsilon+8\right)}{(\varepsilon-2)^{2}(\varepsilon+7)^{3}\left(\varepsilon^{2}+3\right)}, e_{1}=\frac{\varepsilon}{(2-\varepsilon)(\varepsilon+7)}
\end{gathered}
$$

$\Delta(w)=\operatorname{trace}\left(D^{2} w\right)$ being the Laplacian, $S_{2}(w)$ and $S_{4}(w)$ being respectively the second and the forth symmetric functions of the eigenvalues of $D^{2} w$.

Proof. This follows immediately from Lemma 3.1 and a simple calculation since

$$
\Delta(w)=-a_{1, \delta}, S_{2}(w)=a_{2, \delta}, S_{4}(w)=a_{4, \delta}, \operatorname{det}\left(D^{2} w\right)=-a_{5, \delta} .
$$

Let then determine the ordered spectrum $\left\{\lambda_{1}, \lambda_{2}, \ldots, \lambda_{5}\right\}$, $\lambda_{1} \geq \lambda_{2} \geq \ldots \geq \lambda_{5}$ of $D^{2} w$.

\section{Lemma 3.2.}

Let $\lambda_{1} \geq \lambda_{2} \geq \ldots \geq \lambda_{5}$ be the eigenvalues of $D^{2} w_{5, \delta}(x)$. Then

$$
\begin{gathered}
\lambda_{1}=\mu_{2, \delta}, \quad \lambda_{5}=\mu_{3, \delta}, \\
\lambda_{2}= \begin{cases}\mu_{4, \delta} & \text { for } p \in\left[-1, p_{0}(\delta)\right], \\
\mu_{1, \delta} & \text { for } p \in\left[p_{0}(\delta), 1\right],\end{cases} \\
\lambda_{3}= \begin{cases}\mu_{5, \delta} & \text { for } p \in\left[-1,-p_{0}(\delta)\right], \\
\mu_{1, \delta} & \text { for } p \in\left[-p_{0}(\delta), p_{0}(\delta)\right], \\
\mu_{4, \delta} & \text { for } p \in\left[p_{0}(\delta), 1\right],\end{cases} \\
\lambda_{4}= \begin{cases}\mu_{1, \delta} & \text { for } p \in\left[-1,-p_{0}(\delta)\right], \\
\mu_{5, \delta} & \text { for } p \in\left[-p_{0}(\delta), 1\right],\end{cases}
\end{gathered}
$$

where

$$
\left.\left.p_{0}(\delta):=\frac{3^{1 / 4} \sqrt{1-\delta}}{\left(3+2 \delta-\delta^{2}\right)^{1 / 4}}=\frac{3^{1 / 4} \sqrt{\varepsilon}}{\left(4-\varepsilon^{2}\right)^{1 / 4}} \in\right] 0,1\right] .
$$


Proof. The inequalities $\mu_{2, \delta}(p) \geq \mu_{1, \delta}(p) \geq \mu_{3, \delta}(p)$ are obvious since $\mu_{2, \delta}(p)$ and $\mu_{3, \delta}(p)$ are decreasing in $p, \mu_{1, \delta}(p)$ is increasing in $p, \mu_{3, \delta}(-1)=\mu_{1, \delta}(-1)$, $\mu_{2, \delta}(1)=\mu_{1, \delta}(1)$.

The resultant

$$
R(\delta, p)=\operatorname{Res}\left(F_{2}, F_{3}\right)=144(p-1)^{2}(p+1)^{2}\left(r_{8} p^{8}-r_{6} p^{6}+r_{4} p^{4}-r_{2} p^{2}+r_{0}\right)
$$

where

$$
\begin{gathered}
r_{8}=\left(\varepsilon^{2}-4\right)^{2}, r_{6}=12\left(\varepsilon^{2}-4\right)^{2}, r_{4}=3\left(4-\varepsilon^{2}\right)\left(72-17 \varepsilon^{2}\right), \\
r_{2}=108\left(\varepsilon^{2}-4\right)^{2}, r_{0}=144\left(3-\varepsilon^{2}\right)^{2}
\end{gathered}
$$

is strictly positive for $(\varepsilon, p) \in] 0,1[\times]-1,1[$. Indeed, let

$$
r:=\frac{R}{144(p-1)^{2}(p+1)^{2}}=r_{8} p^{8}-r_{6} p^{6}+r_{4} p^{4}-r_{2} p^{2}+r_{0}
$$

then

$d:=\frac{\partial r}{4 \varepsilon \partial \varepsilon}=\left(\varepsilon^{2}-4\right) p^{8}+12 p^{6}\left(4-\varepsilon^{2}\right)+3\left(17 \varepsilon^{2}-70\right) p^{4}+108\left(4-\varepsilon^{2}\right) p^{2}+144\left(\varepsilon^{2}-3\right)<0$

for $(\varepsilon, p) \in] 0,1[\times[0,1[$ since

$$
\frac{\partial d}{4 p \partial p}=\left(4-\varepsilon^{2}\right)\left(-2 p^{6}+18 p^{4}-51 p^{2}+54\right)-6 p^{2} \geq\left(4-\varepsilon^{2}\right) \cdot 19-6 \geq 51,
$$

and for $p=1$ one has $d=-166+76 \varepsilon^{2} \leq-90$. For $\delta=0, \varepsilon=1$ we get

$$
\left.R(\delta, p) \geq R(1, p)=9\left(1-p^{2}\right)\left(4-p^{2}\right)\right)\left(p^{4}-7 p^{2}+16\right)
$$

which proves the positivity. Using then the inequalities

$$
\begin{gathered}
\mu_{2, \delta}(-1)=\mu_{4, \delta}(-1)>\mu_{5, \delta}(-1)>\mu_{3, \delta}(-1), \\
\mu_{2, \delta}(1)>\mu_{4, \delta}(1)>\mu_{5, \delta}(-1)=\mu_{3, \delta}(-1)
\end{gathered}
$$

and the postivity of the resultant we get

$$
\mu_{2, \delta}(p) \geq \mu_{4, \delta}(p) \geq \mu_{5, \delta}(p) \geq \mu_{3, \delta}(p)
$$

for any $p \in[-1,1]$.

Calculatig then

$$
R_{1}(\delta, p)=\operatorname{Res}\left(F_{1}, F_{3}\right)=12\left(p^{2}-3\right)\left(p^{4}\left(\varepsilon^{2}-4\right)+3 \varepsilon^{2}\right)
$$

and taking into account the equalities

$$
\mu_{4, \delta}\left(p_{0}(\delta)\right)=\mu_{1, \delta}\left(p_{0}(\delta)\right), \mu_{5, \delta}\left(-p_{0}(\delta)\right)=\mu_{1, \delta}\left(-p_{0}(\delta)\right)
$$


we get the result.

Note the oddness property of the spectrum:

$$
\lambda_{1, \delta}(-p)=-\lambda_{5, \delta}(p), \lambda_{2, \delta}(-p)=-\lambda_{4, \delta}(p), \lambda_{3, \delta}(-p)=-\lambda_{3, \delta}(p) .
$$

Let us now verify the second condition (2.2) of Lemma 2.1, namely the uniform hyperbolicity of $M_{\delta}(a, b, O)$.

Proposition 3.1. Let $M_{\delta}(x)=D^{2} w_{\delta}(x), 0 \leq \delta<1$. Suppose that $a \neq b \in$ $B_{1} \backslash\{0\}$ and let $O \in O(5)$ be an orthogonal matrix s.t.

$$
M_{\delta}(a, b, O):=M_{\delta}(a)-{ }^{t} O \cdot M_{\delta}(b) \cdot O \neq 0 .
$$

Denote $\Lambda_{1} \geq \Lambda_{2} \geq \ldots \geq \Lambda_{5}$ the eigenvalues of the matrix $M_{\delta}(a, b, O)$. Then

$$
\frac{1}{C} \leq-\frac{\Lambda_{1}}{\Lambda_{5}} \leq C
$$

for $C:=\frac{1000(\delta+1)(3-\delta)}{3(1-\delta)^{2}}$.

Proof. The proof depends on the value of

$$
k:=p_{0}(\delta):=\frac{3^{1 / 4} \sqrt{1-\delta}}{\left(3+2 \delta-\delta^{2}\right)^{1 / 4}}=\frac{3^{1 / 4} \sqrt{\varepsilon}}{\left(4-\varepsilon^{2}\right)^{1 / 4}} .
$$

Note that $C=\frac{1000(\delta+1)(3-\delta)}{3(1-\delta)^{2}}=\frac{1000}{k^{4}}$. We shall give the proof for $\left.\left.k \in\right] 0, \frac{1}{2}\right]$, the proof for $k \in\left[\frac{1}{2}, 1\right]$ is similar, simpler and uses $C=10^{4}$.

Suppose that the conclusion does not hold, that is for some $a \neq b$ and some $O \in \mathrm{O}(5)$ one has

$$
M_{\delta}(a, b, O):=M_{\delta}(a)-{ }^{t} O \cdot M_{\delta}(b) \cdot O \neq 0
$$

but

$$
\frac{1}{C}>-\frac{\Lambda_{1}}{\Lambda_{5}} \text { or }-\frac{\Lambda_{1}}{\Lambda_{5}}>C
$$

We can suppose without loss that $|b| \leq 1=|a| \in \mathbb{S}_{1}^{4}$. Let $\bar{b}:=b /|b| \in \mathbb{S}_{1}^{4}$, $W:=W(a), \bar{W}:=W(\bar{b}), K:=|b|^{-1-\delta}$. Note that since for any harmonic cubic polynomial $Q(x)$ on $\mathbb{R}^{n}$ and any $a \in \mathbb{S}_{1}^{n-1} \subset \mathbb{R}^{n}$ one has

$$
\operatorname{Tr}\left(D^{2}\left(\frac{Q(x)}{|x|^{1+\delta}}\right)(a)\right)=\left(\delta^{2}-2 \delta-3-n\right) Q(a),
$$

we get $\operatorname{Tr}\left(M_{\delta}(a, b, O)\right)=\left(2 \delta+8-\delta^{2}\right)(K \bar{W}-W), P_{5}$ being harmonic. Let us prove the claim for $(K \bar{W}-W) \geq 0$, the proof for $(K \bar{W}-W) \leq 0$ being the same while permuting $a$ with $b$ and $\Lambda_{1}$ with $\Lambda_{5}$. Since

$$
\operatorname{Tr}\left(M_{\delta}(a, b, O)\right)=\left(2 \delta+8-\delta^{2}\right)(K \bar{W}-W) \geq 0,
$$


we get $4 \Lambda_{1}+\Lambda_{5} \geq 0$ and $-\Lambda_{5} / \Lambda_{1} \leq 4$. Therefore, we have only to rule out the inequality $\frac{1}{C}>-\frac{\Lambda_{1}}{\Lambda_{5}}$ i.e. $-\Lambda_{5}>C \Lambda_{1}$. Recall that

$$
W=\frac{3 p-p^{3}}{2}, \bar{W}=\frac{3 \bar{p}-\bar{p}^{3}}{2}
$$

for some $p, \bar{p} \in[-1,1]$.

We have then 3 possibilities:

1). $p, \bar{p} \in[-k, k]$

2). $p \in[-k, k], \bar{p} \notin[-k, k]$;

3). $p, \bar{p} \notin[-k, k]$.

In the cases 1) and 3) applying Lemma 2.4 we get $\Lambda_{1} \geq \mu_{+}(K), \Lambda_{5} \leq \mu_{-}(K)$ in the notation of Lemma 2.5 which permits to finish the proof as in Proposition 4.1 of [NV3]. We thus have to treat the (more difficult) case 2). Lemma 2.4 together with the inequality $-\Lambda_{5}>C \Lambda_{1}$ gives

$$
-\min _{i=1, \cdots, 5}\left\{K \lambda_{i, \delta}(\bar{p})-\lambda_{i, \delta}(p)\right\}>C \max _{i=1, \cdots, 5}\left\{K \lambda_{i, \delta}(\bar{p})-\lambda_{i, \delta}(p)\right\} .
$$

Thank to the oddness of the spectrum we suppose without loss that $\bar{p}>k$. Recall that then by Lemma 3.2 one has

$$
\begin{gathered}
\lambda_{1, \delta}(\bar{p})=\mu_{2, \delta}(\bar{p}), \lambda_{1, \delta}(p)=\mu_{2, \delta}(p), \lambda_{2, \delta}(\bar{p})=\mu_{1, \delta}(\bar{p}), \lambda_{1, \delta}(p)=\mu_{4, \delta}(p), \\
\lambda_{3, \delta}(\bar{p})=\mu_{4, \delta}(\bar{p}), \lambda_{3, \delta}(p)=\mu_{1, \delta}(p), \lambda_{4, \delta}(\bar{p})=\mu_{5, \delta}(\bar{p}), \lambda_{4, \delta}(p)=\mu_{5, \delta}(p), \\
\lambda_{5, \delta}(\bar{p})=\mu_{3, \delta}(\bar{p}), \lambda_{5, \delta}(p)=\mu_{3, \delta}(p) .
\end{gathered}
$$

We have then 2 possibilities for $p$ :

2a). $p \in[-k, 0]$;

2b). $p \in] 0, k]$.

Let $p \in[-k, 0]$, then $\mu_{1, \delta}(p) \leq 0$ and thus

$$
\begin{gathered}
C \max _{i=1, \cdots, 5}\left\{K \lambda_{i, \delta}(\bar{p})-\lambda_{i, \delta}(p)\right\} \geq C K \lambda_{3, \delta}(\bar{p})=C K \mu_{4, \delta}(\bar{p}) \geq C K \mu_{4, \delta}\left(p_{0}(\delta)\right)= \\
=C K \mu_{1, \delta}(k)=C K\left(k^{3}\left(\sqrt{k^{4}+3}-k^{2}+3\right) / \sqrt{k^{4}+3}\right) \geq 2 C K k^{3}
\end{gathered}
$$

since one verifies that the function $\mu_{4, \delta}(p)$ is increasing on $[k, 1]$.

On the other hand,

$\left|\min _{i=1, \cdots, 5}\left\{K \lambda_{i, \delta}(\bar{p})-\lambda_{i, \delta}(p)\right\}\right| \leq K \max _{i=1, \cdots, 5, p}\left|\left\{\lambda_{i, \delta}(p)\right\}\right|+\max _{i=1, \cdots, 5, p}\left|\left\{\lambda_{i, \delta}(p)\right\}\right| \leq 8(K+1)$.

Therefore one gets $8(K+1) \geq 2 C K k^{3}$ which clearly is a contradiction for, say, $K \geq 1 / 4$. For $0<K \leq 1 / 4$ we get

$C \max _{i=1, \cdots, 5}\left\{K \lambda_{i, \delta}(\bar{p})-\lambda_{i, \delta}(p)\right\} \geq C\left(K \lambda_{5, \delta}(\bar{p})-\lambda_{5, \delta}(p)\right) \geq C(K(-8)-(-5)) \geq 3 C$ 
which can not be less than $8(K+1) \leq 10$.

Let finally $p \in] 0, k]$. We consider then 2 possibilities for $K$ :

(i) $K \leq 20 / 31=(1.55)^{-1}$,

(ii) $K>20 / 31=(1.55)^{-1}$.

In the case $(i)$ one has

$$
\begin{gathered}
C \max _{i=1, \cdots, 5}\left\{K \lambda_{i, \delta}(\bar{p})-\lambda_{i, \delta}(p)\right\} \geq C\left(K \lambda_{5, \delta}(\bar{p})-\lambda_{5, \delta}(p)\right) \geq \\
\geq C\left(K \mu_{3, \delta}(1)-\mu_{3, \delta}(0)\right) \geq C(3 \sqrt{3}+20(\varepsilon-8) / 31)>C / 30>8(K+1)
\end{gathered}
$$

since $\lambda_{5, \delta}(p)=\mu_{3, \delta}(p)$ is decreasing, $\mu_{3, \delta}(0)=-3 \sqrt{3}, \mu_{3, \delta}(1)=\varepsilon-8$.

We suppose then $K>20 / 31=(1.55)^{-1}$. Then if $p \leq 3 k / 4$ one has

$$
\begin{gathered}
C \max _{i=1, \cdots, 5}\left\{K \lambda_{i, \delta}(\bar{p})-\lambda_{i, \delta}(p)\right\} \geq C K\left(\lambda_{3, \delta}(\bar{p})-\lambda_{3, \delta}(p) / K\right) \geq C K\left(\mu_{4, \delta}(\bar{p})-\mu_{1, \delta}(p) / K\right) \geq \\
\geq C K\left(\mu_{4, \delta}(k)-\mu_{1, \delta}(3 k / 4) / K\right)=C K\left(\mu_{1, \delta}(k)-\mu_{1, \delta}(3 k / 4) / K\right)> \\
>C K \mu_{1, \delta}\left(\frac{3 k}{4}\right)\left(\frac{\left.\mu_{4, \delta}(k)\right)}{\mu_{1, \delta}\left(\frac{3 k}{4}\right)}-\frac{31}{20}\right) \geq \\
\geq C K \frac{\mu_{1, \delta}\left(\frac{3 k}{4}\right)}{100} \geq C K \frac{2 k^{3}}{100}>20 K>8(K+1),
\end{gathered}
$$

contradiction for $k \in[0,1 / 2]$ since $\frac{\left.\mu_{4, \delta}(k)\right)}{\mu_{1, \delta}\left(\frac{3 k}{4}\right)}>1.56, \mu_{1, \delta}\left(\frac{3 k}{4}\right)>2 k^{3}$ there. Thus we can suppose that $\left.p \in] \frac{3 k}{4}, k\right]$. One notes then that $\mu_{4, \delta}(p) \leq \mu_{4, \delta}(k)$ for $p \in\left[\frac{k}{4}, 1\right]$. This permits to rule out the case $\bar{p} \geq \frac{3 k}{2}$. Indeed, one has in this case

$$
\begin{gathered}
C \max _{i=1, \cdots, 5}\left\{K \lambda_{i, \delta}(\bar{p})-\lambda_{i, \delta}(p)\right\} \geq C K\left(\lambda_{2, \delta}(\bar{p})-\lambda_{2, \delta}(p) / K\right) \geq C K\left(\mu_{1, \delta}(\bar{p})-\mu_{4, \delta}(p) / K\right) \geq \\
\geq C K\left(\mu_{1, \delta}(3 k / 2)-\mu_{1, \delta}(k) / K\right)=C K\left(\mu_{1, \delta}(3 k / 2)-\mu_{1, \delta}(k) / K\right),
\end{gathered}
$$

and one gets a contradiction as above since

$$
\frac{\mu_{1, \delta}\left(\frac{3 k}{2}\right)}{\mu_{1, \delta}(k)}>2
$$

for $k \in[0,1 / 2]$.

The last case to rule out is thus $K \geq 20 / 31, p \in\left[\frac{3 k}{4}, k\right], \bar{p} \in\left[k, \frac{3 k}{2}\right]$. Let then

$$
\alpha:=k-p \in\left[0, \frac{k}{4}\right] \subset\left[0, \frac{1}{8}\right], \beta:=\bar{p}-k \in\left[0, \frac{k}{2}\right] \subset\left[0, \frac{1}{4}\right], a:=\max \{\alpha, \beta\} .
$$

It is easy to verify that on $\left[\frac{3 k}{4}, \frac{3 k}{2}\right]$ one has the following inequalities:

$$
\frac{\partial \mu_{1, \delta}(p)}{\partial p} \geq 3 k^{2}, \frac{11 k^{3}}{4} \geq \mu_{1, \delta}(k) \geq \frac{5 k^{3}}{2} ;
$$




$$
\begin{gathered}
\frac{\partial \mu_{2, \delta}(p)}{\partial p} \geq-5,4 k-5 \geq \mu_{2, \delta}(k) \geq 4 k-\frac{11}{2} ; \\
\frac{\partial \mu_{3, \delta}(p)}{\partial p} \geq-\frac{9}{2},-5-3 k \geq \mu_{3, \delta}(k) \geq-\frac{11+7 k}{2} ; \\
\frac{\partial \mu_{4, \delta}(p)}{\partial p} \geq-\frac{k}{29}, \frac{11 k^{3}}{4} \geq \mu_{4, \delta}(k) \geq \frac{5 k^{3}}{2} ; \\
\frac{\partial \mu_{5, \delta}(p)}{\partial p} \geq 10 k^{2}-12,-10 k \geq \mu_{5, \delta}(k) \geq-12 k .
\end{gathered}
$$

Let then $K \in\left[\frac{20}{31}, 1\right]$. Therefore,

$$
\begin{gathered}
C \max _{i=1, \cdots, 5}\left\{K \lambda_{i, \delta}(\bar{p})-\lambda_{i, \delta}(p)\right\} \geq \\
\geq C \max \left\{K \mu_{1, \delta}(k+\alpha)-\mu_{1, \delta}(k-\beta), K \mu_{3, \delta}(k+\alpha)-\mu_{3, \delta}(k-\beta)\right\} \geq \max \left\{M_{1}, M_{2}\right\}= \\
=C \max \left\{3(K-1) k^{3} a+3(K+1) k^{2},(1-K)(5+3 k) a-\frac{(11+7 k)(K+1)}{10}\right\}
\end{gathered}
$$

for linear forms $M_{1}, M_{2}$ in $K$. Note that the minimal value of $\max \left\{M_{1}, M_{2}\right\}$ as a function of $K$ equals (recall that our $C=1000 / k^{4}$ ):

$$
\frac{1500 a(40-9 k)}{k^{2}\left(12 k^{2} a+18 a+11 k^{3}+20+12 k\right)}>\frac{1250 a}{k^{2}}>0
$$

attained for $K=K_{0}:=\left(11 k^{3}+20+12 k\right) /\left(12 k^{2} a+18 a+11 k^{3}+20+12 k\right)<1$.

On the other hand,

$$
-\Lambda_{5} \leq-\min _{i=1, \cdots, 5}\left\{K \lambda_{i, \delta}(\bar{p})-\lambda_{i, \delta}(p)\right\} \leq \max \left\{l_{1}, l_{2}, l_{3}, l_{4}, l_{5}\right\}
$$

for the following linear forms (in $K$ )

$$
\begin{gathered}
l_{1}:=-k^{2}\left(\frac{11 a}{4}+3 k\right) K-\frac{11 a}{4} k^{2}+3 k^{3}, \\
l_{2}:=\left(5 a+4 k-\frac{11}{2}\right) K+5 a+\frac{11}{2}-4 k, \\
l_{3}:=(5 a+5+3 k) K+5 a-5-3 k, \\
l_{4}:=\left(\frac{a k}{29}-\frac{11 k^{3}}{2}\right) K+\frac{a k}{29}+\frac{11 k^{3}}{2}, \\
l_{5}:=\left(\left(12-10 k^{2}\right) a+12 k\right) K+\left(12-10 k^{2}\right) a-12 k .
\end{gathered}
$$

To refute our inequality it is sufficient to prove that $M_{i}\left(K_{j, k}\right)>0$ for any triple $(i, j, k)$ with $i, j \in\{1,2\}, i \neq j, k \in\{1,2,3,4,5\}$ where $l_{k}\left(K_{j, k}\right)=M_{j}\left(K_{j, k}\right)$.

Explicit calculations give (for the values $m_{i j k}:=\frac{M_{i}\left(K_{j, k}\right)}{500 a k^{2}}$ )

$$
\frac{m_{121}}{3}=\frac{\left(9 k^{4}+6 k^{6}\right) a+10000+5 k^{7}+20 k^{4}+3 k^{5}-2250 k}{k^{2}\left(\left(3 k^{4}+3000\right) a+3 k^{5}+2750 k\right)}>0,
$$




$$
\begin{aligned}
& \frac{m_{211}}{3}=\frac{\left(9 k^{4}+6 k^{6}\right) a+10000+5 k^{7}+20 k^{4}+3 k^{5}-2250 k}{\left(4500-3 k^{6}\right) a+5000+3000 k-3 k^{7}}>0, \\
& m_{122}=\frac{60000-\left(60 k^{4}+90 k^{2}\right) a-192 k^{3}+66 k^{4}-13500 k-101 k^{2}-158 k^{5}}{k^{2}\left(\left(6000-10 k^{2}\right) a-11 k^{2}+8 k^{3}+5500 k\right)}>0, \\
& m_{212}=\frac{60000-\left(60 k^{4}+90 k^{2}\right) a-192 k^{3}+66 k^{4}-13500 k-101 k^{2}-158 k^{5}}{\left(10 k^{4}+9000\right) a+11 k^{4}-8 k^{5}+10000+6000 k}>0 \text {, } \\
& m_{123}=\frac{30000-\left(45 k^{2}+30 k^{4}\right) a-55 k^{2}-6750 k-33 k^{3}+30 k^{4}-37 k^{5}}{k^{2}\left(\left(3000-5 k^{2}\right) a+2750 k-5 k^{2}-3 k^{3}\right)}>0, \\
& m_{213}=\frac{30000-\left(45 k^{2}+30 k^{4}\right) a-55 k^{2}-6750 k-33 k^{3}+30 k^{4}-37 k^{5}}{\left(5 k^{4}+4500\right) a+5 k^{4}+3 k^{5}+5000+3000 k}>0, \\
& m_{124}=\frac{3480000-\left(36 k^{3}+24 k^{5}\right) a-t(k)}{k^{2}\left(\left(348000-4 k^{3}\right) a+319000 k+319 k^{5}\right)}>0, \\
& m_{214}=\frac{3480000-\left(36 k^{3}+24 k^{5}\right) a-t(k)}{\left(522000+4 k^{5}\right) a+580000+348000 k-319 k^{7}}>0, \\
& m_{215}=\frac{\left(9 k^{4}+30 k^{6}-54 k^{2}\right) a+15000-u(k)}{5 k^{4} a+1500 a-6 k^{3}+1375 k-6 k^{2} a}>0, \\
& m_{125}=\frac{\left(9 k^{4}+30 k^{6}-54 k^{2}\right) a+15000-u(k)}{\left(2250+6 k^{4}-5 k^{6}\right) a+2500+1500 k+6 k^{5}}>0,
\end{aligned}
$$

Let, finally $K \geq 1$, then

$$
\begin{gathered}
C \Lambda_{1} \geq C\left(K \mu_{1, \delta}(k+\alpha)-\mu_{1, \delta}(k-\beta)\right) \geq \frac{5 C}{2}(K-1) k^{3} a+3(K+1) C k^{2}= \\
=L_{1}:=\frac{500(5 k K+6 a-5 k)}{k^{2}}=\frac{2500(K-1)}{k}+\frac{3000 a}{k^{2}},
\end{gathered}
$$

and

$$
-\Lambda_{5} \leq-\min _{i=2, \cdots, 5}\left\{K \lambda_{i, \delta}(\bar{p})-\lambda_{i, \delta}(p)\right\} \leq \max \left\{L_{2}, L_{3}, L_{4}, L_{5}\right\}
$$

for the following linears forms in $K$ :

$$
\begin{aligned}
L_{2} & :=5(K+1) a+(1-K)(5-4 k)=(5 a-5+4 k)(K-1)+10 a, \\
L_{3} & :=\frac{9}{2}(K+1) a+\frac{11+7 k}{2}(K-1)=\frac{9 a+11+7 k}{2}(K-1)+9 a, \\
L_{4} & :=(K+1) a \frac{k}{29}-\frac{5 k^{3}}{2}(K-1)=\left(\frac{a k}{29}-\frac{5 k^{3}}{2}\right)(K-1)+\frac{2 a k}{29}, \\
L_{5} & :=12 k(K+1) a+\frac{9}{2}(K-1)=3\left(4 a k+\frac{3}{2}\right)(K-1)+24 a k .
\end{aligned}
$$


One immediately sees that both the slope and the value at $K=1$ of $L_{1}$ are (much) bigger than those of $L_{i}, i=2,3,4,5$ which finishes the proof.

Proof of Theorem 1.1. To prove the result it is sufficient to verify the condition (2.1) in Lemma 2.1, namely, that the five partial derivatives $\frac{\partial g}{\partial \lambda_{i}}, i=1, \ldots, 5$ are strictly positive (and bounded which is automatic thank to compacity) on the symmetrized image

$$
M:=\bigcup_{\sigma \in \Sigma_{n}} T_{\sigma} \Lambda(B) \subset \mathbb{R}^{n}
$$

of the unit ball under the map $\Lambda$,

$$
g\left(\lambda_{1}, \ldots, \lambda_{5}\right)=\operatorname{det}\left(D^{2} w\right)-e_{5}(\Delta(w))^{5}-e_{3}(\Delta(w))^{3} S_{2}(w)-e_{1} \Delta(w) S_{4}(w)
$$

being our equation. By homogeneity it is sufficient to show this on $M^{\prime}:=\Lambda\left(\mathbb{S}_{1}^{4}\right)$ which is an algebraic curve, the union of 120 curves $T_{\sigma} \Lambda\left(\mathbb{S}_{1}^{4}\right)$ and that it is sufficient, by symmetry, to verify the condition on the curve $\Lambda\left(\mathbb{S}_{1}^{4}\right)$ only. A brute force calculation shows then that

$$
\begin{gathered}
g_{1}(p, \varepsilon):=\frac{\partial g}{\partial \lambda_{1}}=\sum_{i=0}^{12} m_{i} p^{i}= \\
=m_{12} p^{8}\left(p^{4}-12 p^{2}+54\right)+m_{9} b^{3}+m_{6} p^{4}\left(p^{2}-\frac{3}{4}\right)+m_{2}+m_{0},
\end{gathered}
$$

with

$$
\begin{gathered}
m_{12}=3\left(\varepsilon^{4}+3 \varepsilon^{3}-20 \varepsilon^{2}+12 \varepsilon-56\right)(\varepsilon-2)^{2}(\varepsilon+2)^{2}, m_{10}=-12 m_{12}, m_{8}=54 m_{12}, \\
m_{11}=m_{1}=0, m_{9}=3 D(p, \varepsilon)(\varepsilon+7)(\varepsilon+2)\left(\varepsilon^{2}+2\right)(\varepsilon-2)^{2}, m_{7}=-9 m_{9}, m_{5}=27 m_{9}, \\
m_{6}=108(2-\varepsilon)\left(3 \varepsilon^{7}+17 \varepsilon^{6}-54 \varepsilon^{5}-152 \varepsilon^{4}+72 \varepsilon^{3}-42 \varepsilon^{2}+384 \varepsilon+1344\right), m_{3}=27 m_{9}, \\
m_{4}=-\frac{3 m_{6}}{4}, \quad m_{2}=1944 \varepsilon^{2}(2-\varepsilon)\left(\varepsilon^{2}-7\right)\left(\varepsilon^{2}+3\right), \quad m_{0}=-7776 \varepsilon^{2}\left(\varepsilon^{2}+3\right)
\end{gathered}
$$

for $D(p, \varepsilon):=\sqrt{\left(16-\varepsilon^{2}\right)\left(4-\varepsilon^{2}\right) b^{2}+144 \varepsilon^{2}}, b=\left(p^{2}-3\right) p$;

$$
g_{2}(p, \varepsilon):=\frac{\partial g}{\partial \lambda_{2}}=\sum_{i=0}^{12} n_{i} p^{i}
$$

with

$$
\begin{gathered}
n_{12}=(\varepsilon+4)(\varepsilon+1)\left(4-\varepsilon^{2}\right)^{2}, n_{10}=-(\varepsilon+2)\left(\varepsilon^{4}+19 \varepsilon^{3}+86 \varepsilon^{2}+182 \varepsilon+96\right)(2-\varepsilon)^{2}, \\
n_{11}=n_{1}=0, \quad n_{8}=9(\varepsilon+2)\left(\varepsilon^{2}+10 \varepsilon+6\right)\left(\varepsilon^{2}+3 \varepsilon+8\right)(2-\varepsilon)^{2}, \\
n_{9}=\varepsilon(\varepsilon+7)(\varepsilon+2)\left(\varepsilon^{2}+2\right)(2-\varepsilon)^{2} \sqrt{3\left(4-p^{2}\right)}, n_{7}=-9 n_{9}, n_{5}=27 n_{9}, \\
n_{6}=3(2-\varepsilon)\left(13 \varepsilon^{6}+115 \varepsilon^{5}+218 \varepsilon^{4}+170 \varepsilon^{3}-876 \varepsilon^{2}-2856 \varepsilon-1152\right),
\end{gathered}
$$




$$
\begin{gathered}
n_{4}=9(\varepsilon-2)\left(11 \varepsilon^{6}+62 \varepsilon^{5}+33 \varepsilon^{4}-24 \varepsilon^{3}-348 \varepsilon^{2}-1176 \varepsilon-288\right) \\
n_{3}=\varepsilon^{3}(2-\varepsilon)(\varepsilon+7)\left(3 \varepsilon^{2}+2\right) \sqrt{3\left(4-p^{2}\right)} \\
n_{2}=108 \varepsilon^{2}(2-\varepsilon)\left(\varepsilon^{2}+3\right)\left(\varepsilon^{2}+4 \varepsilon-3\right), n_{0}=1296 \varepsilon^{2}\left(\varepsilon^{2}+3\right) \\
g_{3}(p, \varepsilon):=\frac{\partial g}{\partial \lambda_{3}}=\sum_{i=0}^{6} h_{2 i} p^{2 i}
\end{gathered}
$$

with

$$
\begin{gathered}
h_{12}=(\varepsilon+4)(\varepsilon+1)\left(4-\varepsilon^{2}\right)^{2}, h_{10}=2(\varepsilon+2)\left(\varepsilon^{4}+\varepsilon^{3}-40 \varepsilon^{2}-70 \varepsilon-48\right)(2-\varepsilon)^{2} \\
h_{8}=-18(\varepsilon+2)\left(\varepsilon^{4}+4 \varepsilon^{3}-19 \varepsilon^{2}-28 \varepsilon-24\right)(2-\varepsilon)^{2}, \\
h_{6}=6(\varepsilon-2)\left(7 \varepsilon^{6}+37 \varepsilon^{5}-136 \varepsilon^{4}-274 \varepsilon^{3}+330 \varepsilon^{2}+672 \varepsilon+576\right), \\
h_{4}=9(\varepsilon-2)\left(2 \varepsilon^{6}-\varepsilon^{5}+27 \varepsilon^{4}-66 \varepsilon^{3}-348 \varepsilon^{2}-1176 \varepsilon-288\right), \\
h_{2}=108 \varepsilon(2-\varepsilon)\left(\varepsilon^{3}+4 \varepsilon^{2}-15 \varepsilon-84\right)\left(\varepsilon^{2}+3\right), \\
h_{0}=-1296 \varepsilon\left(\varepsilon^{2}+3\right)\left(\varepsilon^{2}+4 \varepsilon-14\right) ; \\
g_{4}(p, \varepsilon):=\frac{\partial g}{\partial \lambda_{4}}=g_{1}(-p, \varepsilon), \\
g_{5}(p), \varepsilon:=\frac{\partial g}{\partial \lambda_{5}}=g_{2}(-p, \varepsilon),
\end{gathered}
$$

and thus we need to consider only the functions $g_{1}(p, \varepsilon), g_{2}(p, \varepsilon), g_{3}(p, \varepsilon)$ on the set $[-1,1] \times(0,1]$. We have to prove that for any fixed $\varepsilon \in(0,1]$ they are strictly positive.

The technique of the proof is identical for all three derivatives, and we begin with $g_{3}$ wich is slightly simpler since it is a polynomial in two variables. One can rearrange it in the form

$$
g_{3}(p, \varepsilon)=g_{37} \varepsilon^{7}+g_{36} \varepsilon^{6}+g_{35} \varepsilon^{5}+g_{34} \varepsilon^{4}+g_{33} \varepsilon^{3}+g_{32} \varepsilon^{2}+g_{31} \varepsilon+g_{30}
$$

with

$$
\begin{gathered}
g_{37}=2 q^{5}+42 q^{3}-18 q^{4}-108 q+18 q^{2}, g_{36}=138 q^{3}-2 q^{5}-216 q+q^{6}-36 q^{4}-45 q^{2}, \\
g_{35}=-92 q^{5}+558 q^{4}+2160 q+5 q^{6}-1296-1260 q^{3}+261 q^{2} \\
g_{34}=-4 q^{6}+5184 q-12 q^{3}-5184-1080 q^{2}+28 q^{5}-36 q^{4} \\
g_{33}=-1944 q^{2}-2520 q^{4}-40 q^{6}+14256-10692 q+5268 q^{3}+520 q^{5} \\
g_{32}=-144 q^{4}+72 q^{3}+112 q^{5}+17496 q-4320 q^{2}-16 q^{6}-15552, \\
g_{31}=-736 q^{5}+80 q^{6}+2304 q^{4}-54432 q+54432-4608 q^{3}+18576 q^{2}, g_{30}=64 q^{2}(q-3)^{4} \geq 0
\end{gathered}
$$


for $q=p^{2} \in[0,1]$. Therefore,

$$
g_{3}(p, \varepsilon) \geq \varepsilon\left(\bar{g}_{37} \varepsilon^{6}+\bar{g}_{36} \varepsilon^{5}+\bar{g}_{35} \varepsilon^{4}+\bar{g}_{34} \varepsilon^{3}+\bar{g}_{33} \varepsilon^{2}+\bar{g}_{32} \varepsilon+\bar{g}_{31}\right)
$$

where $\bar{g}_{3 i}:=\min _{q \in[0,1]} g_{3 i}(q)$, and an elementary calculation gives

$$
\frac{g_{3}(p, \varepsilon)}{\varepsilon} \geq-64 \varepsilon^{5}-160 \varepsilon^{4}-5184 \varepsilon^{3}+4848 \varepsilon^{2}-15552 \varepsilon+15616>1620
$$

for $\varepsilon \in\left(0, \frac{9}{10}\right]$. For $\varepsilon \in\left[\frac{9}{10}, 1\right]$ we have $g_{3}(p, \varepsilon) \geq \sum_{i=0}^{6} \bar{h}_{2 i} q^{i}$ where $\bar{h}_{2 i}:=$ $\min _{\varepsilon \in\left[\frac{9}{10}, 1\right]} h_{2 i}$ and thus

$g_{3}(p, \varepsilon) \geq-736 q^{5}+80 q^{6}+2304 q^{4}-54432 q+54432-4608 q^{3}+18576 q^{2}>4840$.

Thus, finally $g_{3}(p, \varepsilon) \geq \min \{1620 \varepsilon, 4840\}$.

The function $g_{1}(p, \varepsilon)=s_{1}-t_{1}$ with

$$
\begin{gathered}
s_{1}:=\left(q^{3}-6 q^{2}+9 q\right) \varepsilon^{7}+\left(5 q^{3}-30 q^{2}+45 q-72\right) \varepsilon^{6}+\left(108 q^{2}-18 q^{3}-162 q\right) \varepsilon^{5}+ \\
\left(-432 q+288-48 q^{3}+288 q^{2}\right) \varepsilon^{4}+\left(24 q^{3}-144 q^{2}+216 q\right) \varepsilon^{3}+ \\
1512 \varepsilon^{2}+\left(1152 q-768 q^{2}+128 q^{3}\right) \varepsilon+448 q^{2}(q-3)^{4} \geq \\
-72 \varepsilon^{6}-72 \varepsilon^{5}+96 \varepsilon^{4}+1512 \varepsilon^{2} \geq 1440 \varepsilon^{2}>0
\end{gathered}
$$

and

$$
t_{1}:=\left(\varepsilon^{2}-4\right)(\varepsilon+7)\left(\varepsilon^{2}+2\right) b D(p, \varepsilon) ;
$$

simplifying $s_{1}^{2}-t_{1}^{2}=\left(s_{1}-t_{1}\right)\left(s_{1}+t_{1}\right)=g_{1}(p, \varepsilon)\left(s_{1}+t_{1}\right)$ one finds

$$
\begin{gathered}
\left(540 q^{2}-1296 q-216 q^{4}-4 q^{6}+288 q^{3}+48 q^{5}\right) \varepsilon^{13}+ \\
\left(-1944 q^{4}+3024 q^{3}+432 q^{5}-7776 q+5184+2268 q^{2}-36 q^{6}\right) \varepsilon^{12}+ \\
\left(-2052 q^{2}+3240 q^{4}-5328 q^{3}-720 q^{5}+60 q^{6}+10368 q\right) \varepsilon^{11}+ \\
\left(936 q^{6}+50544 q^{4}+55944 q^{2}-11232 q^{5}-41472-97776 q^{3}+29808 q\right) \varepsilon^{10} \\
+\left(26136 q^{2}+120 q^{6}-15696 q^{3}+6480 q^{4}-24624 q-1440 q^{5}\right) \varepsilon^{9}+ \\
\left(57456 q^{5}+156816 q-492372 q^{2}-4788 q^{6}-134784+534528 q^{3}-258552 q^{4}\right) \varepsilon^{8}+ \\
\left(180576 q^{2}-352 q^{6}-313632 q+3168 q^{3}+4224 q^{5}-19008 q^{4}\right) \varepsilon^{7}+ \\
\left(54432 q^{4}-238464 q^{3}+859248 q^{2}-1166400 q+1008 q^{6}+870912-12096 q^{5}\right) \varepsilon^{6}+ \\
\left(1118592 q^{3}-518400 q^{4}-9600 q^{6}-1268352 q^{2}+736128 q+115200 q^{5}\right) \varepsilon^{5}+ \\
\left(1524096 q^{4}+207360 q+2286144+28224 q^{6}-338688 q^{5}+2147904 q^{2}-3025152 q^{3}\right) \varepsilon^{4}+ \\
\left(10752 q^{6}+580608 q^{4}-903168 q^{3}-677376 q^{2}+2322432 q-129024 q^{5}\right) \varepsilon^{3}+ \\
\left(3640320 q^{3}-25344 q^{6}+304128 q^{5}-1368576 q^{4}+8128512 q-7471872 q^{2}\right) \varepsilon^{2}+ \\
\left(3096576 q^{4}+4644864 q^{2}+57344 q^{6}-688128 q^{5}-6193152 q^{3}\right) \varepsilon \geq
\end{gathered}
$$


$64 \varepsilon^{4}\left(-10 \varepsilon^{9}+18 \varepsilon^{8}-648 \varepsilon^{6}-141 \varepsilon^{5}-2214 \varepsilon^{4}-2266 \varepsilon^{3}+5760 \varepsilon^{2}+35721\right) \geq 2.2 \cdot 10^{6} \varepsilon^{4}$.

Since $s_{1}+t_{1} \leq 10^{6}$ one gets $g_{1}(p, \varepsilon)>2 \varepsilon^{4}$.

Similarly, $g_{2}(p, \varepsilon)=s_{2}-t_{2}$ with a polynomial $s_{2} \geq 3000 \varepsilon^{2}$ and

$$
t_{2}=\varepsilon(\varepsilon+7)(\varepsilon-2) t(\varepsilon, q) p^{3} \sqrt{3(4-q)}
$$

where

$t(\varepsilon, q):=\left(\varepsilon^{4}-2 \varepsilon^{2}-8\right) q^{3}+9\left(-\varepsilon^{4}+2 \varepsilon^{2}+8\right) q^{2}+27\left(\varepsilon^{4}-2 \varepsilon^{2}-8\right) p^{2}-9\left(3 \varepsilon^{2}+2\right) \varepsilon^{2}$.

Simplifying $s_{2}^{2}-t_{2}^{2}=g_{2}(p, \varepsilon)\left(s_{2}+t_{2}\right)$ one gets a polynomial $\geq$

$\left(-2560 \varepsilon^{9}-18176 \varepsilon^{8}-325632 \varepsilon^{6}-1254656 \varepsilon^{4}+2202112 \varepsilon^{2}+15116544\right) \varepsilon^{4} \geq 1.5 \cdot 10^{7} \varepsilon^{4}$

and $g_{2}(p, \varepsilon) \geq 15 \varepsilon^{4}$ which finishes the proof.

\section{REFERENCES}

[C] L. Caffarelli, Interior a priory estimates for solutions of fully nonlinear equations, Ann. Math. 130 (1989), 189-213.

[CC] L. Caffarelli, X. Cabre, Fully Nonlinear Elliptic Equations, Amer. Math. Soc., Providence, R.I., 1995.

[CIL] M.G. Crandall, H. Ishii, P-L. Lions, User's guide to viscosity solutions of second order partial differential equations, Bull. Amer. Math. Soc. (N.S.), 27(1) (1992), 1-67.

[CNS] L. Caffarelli, L. Nirenberg, J. Spruck, The Dirichlet problem for nonlinear second order elliptic equations III. Functions of the eigenvalues of the Hessian, Acta Math. 155 (1985), 261-301.

[F] A. Friedman, Differential games, Pure and Applied Mathematics, vol. 25, John Wiley and Sons, New York, 1971.

[NTV] N. Nadirashvili, V. Tkachev, S. Vlăduţ, A non-classical Solution to Hessian Equation from Cartan Isoparametric Cubic, Adv. Math. 231 (2012), 15891597.

[NV1] N. Nadirashvili, S. Vlăduţ, Nonclassical solutions of fully nonlinear elliptic equations, Geom. Func. An. 17 (2007), 1283-1296.

[NV2] N. Nadirashvili, S. Vlăduț, Singular Viscosity Solutions to Fully Nonlinear Elliptic Equations, J. Math. Pures Appl., 89 (2008), 107-113.

[NV3] N. Nadirashvili, S. Vlăduţ, Octonions and Singular Solutions of Hessian Elliptic Equations, Geom. Func. An. 21 (2011), 483-498.

[NV4] N. Nadirashvili, S. Vlăduț, Singular solutions of Hessian fully nonlinear elliptic equations, Adv. Math. 228 (2011), 1718-1741. 
[NV5] N. Nadirashvili, S. Vlăduţ, Homogeneous Solutions of Fully Nonlinear Elliptic Equations in Four Dimensions, arXiv:1201.1022, to appear in Comm. Pure Appl. Math.

[T1] N. Trudinger, Weak solutions of Hessian equations, Comm. Part. Diff. Eq. 22 (1997), 1251-1261.

[T2] N. Trudinger, On the Dirichlet problem for Hessian equations, Acta Math. 175 (1995), 151-164.

[T3] N. Trudinger, Hölder gradient estimates for fully nonlinear elliptic equations, Proc. Roy. Soc. Edinburgh Sect. A 108 (1988), 57-65. 